\title{
WHY A FUTURE COMMERCIAL SPACECRAFT MUST BE ABLE TO SWIM
}

\author{
Frank Morlang ${ }^{(1)}$, Jorge Ferrand $^{(2)}$, Remzi Seker ${ }^{(2)}$ \\ ${ }^{(1)}$ German Aerospace Center DLR, Lilienthalplatz 7, 38108 Braunschweig, Germany, frank.morlang@dlr.de \\ (2) Embry-Riddle Aeronautical University ERAU, Daytona Beach, FL 32114, USA, ferrandj@erau.edu
}

\begin{abstract}
Regarding current as well as future commercial space transportation (CST) projects, new re-entry trajectory profiles, differing from capsule and shuttle-like approaches, have to be taken into account. These characteristics are mainly ruled by the principle of "landing like an aircraft at an airport". This directly generates the need to be taken in a system wide information management (SWIM) consideration because of the fact, that all the future air traffic participants are requested to act as SWIM communicating sub-systems by the future Single European Sky Air Traffic Management Research (SESAR) SWIM "Intranet for ATM" concept. Against the background of the global character of future CST operations and the associated SWIM harmonization need referring the U.S. Next Generation Air Transportation System (NextGen) and SESAR, the presented solution addresses near real-time information sharing for normal air traffic protection purposes in the event of a future space vehicle breakup and bases on the already harmonized data format standards Aeronautical Information Exchange Model (AIXM) and Flight Information Exchange Model (FIXM).
\end{abstract}

\section{INTRODUCTION}

The concept of SWIM can roughly be condensed to the ability of delivering commonly understood quality information at the right time to the right target(s), resulting in a continuous data flow of the right sources to the right sinks. Strictly following this elemental request, generates the need that future commercial spacecraft will have to obey to it, especially if the generation and distribution of emergency information is concerned, in order to build a fundament for seamless integration into normal air traffic $[6,7]$.

\section{THE VALUE PROPOSITION}

Preparing for the advent of Commercial Space and its impact to standard Air travel requires a Global, Enterprise-level approach that maximizes nonnegotiable Safety and Cyber Security needs while addressing business and economic pragmatics. The approach presented within this Use Case leverages existing international data standards and infrastructures i.e., SWIM. This Use Case clearly demonstrates International Harmonization of Global Airspace to ensure key, critical benefits:
- All data exchanged is from a trusted, secure and authenticated source.

- All airspace stakeholders have shared situational awareness for real-time nominal and emergency situations - this is a globally available solution.

- Policies and Processes to be developed will use data from this approach for coordinated Collaborative Decision Making (CDM).

- Stakeholder notification, dynamic CDM and reconfiguration of the airspace back to a nominal state is achieved using this approach for safe and cost-effective airspace management.

\section{THE CHALLENGE}

Looking at the risk of falling debris from a spacecraft's in-flight explosion or breakup event, a large area can be covered by resulting fragments, depending on the position and velocity of the vehicle in the moment of disintegration. On the one hand, it must be assured that such a hazard area can be cleared of civilian/commercial air traffic in time, on the other hand, airspace segregation needs to be minimal in time and size, enabling an economic and efficient joint air- and space traffic operation. Facing this, the distribution of potential hazard area position information at the right time, to the right people is essential, for efficient flight planning purposes as well as for rapid and correct decisions in case of a fatal event. The optimization of the size and machine-aided decision-making aspects of this project are beyond the scope of this paper and will be handled as future studies as our research progresses.

\section{THE SOLUTION}

\subsection{General Architecture}

The solution's general architecture is shown in Figure 1. It consists of a data server implemented as a Hypertext Transfer Protocol Secure (HTTPS) web server and a provisional hazard area model assuming a Space Shuttle like trajectory of the hypothetical spacecraft. The data server is represented by an HTTPS web server, doing Simple Object Access Protocol (SOAP) message parsing for incoming requests and SOAP message creation for its responses. The provisional hazard area model is realized as an inter-/extrapolation model based on the United States National Aeronautics and Space 
Administration's (NASA) Columbia space shuttle accident debris data [1].

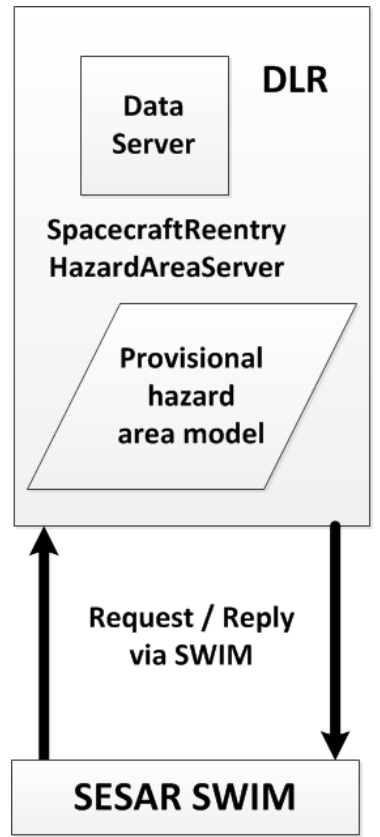

Figure 1. General architecture of the SpacecraftReentryHazardAreaServer

\subsection{SESAR SWIM / FAA SWIM Use Case}

The principle of SESAR SWIM / FAA SWIM demonstration use case is presented in Figure 2. It shows the connection to the United States Federal Aviation Administration's (FAA) concept of Global Enterprise Messaging Service (GEMS), where Service by NEAR (SBN) is one representational implementation of a GEMS provided by the Next Generation ERAU Applied Research (NEAR) lab. The demonstration use case includes Flight Object data exchange using FIXM version 3.0.1 between an FAA research and development Flight Object Exchange Service, an airline EFB, and the SpacecraftReentryHazardAreaService, consuming the aircraft state data within the Flight Object FIXM message and publishes its output using AIXM version 5.1 for consumption and display by other interested parties.

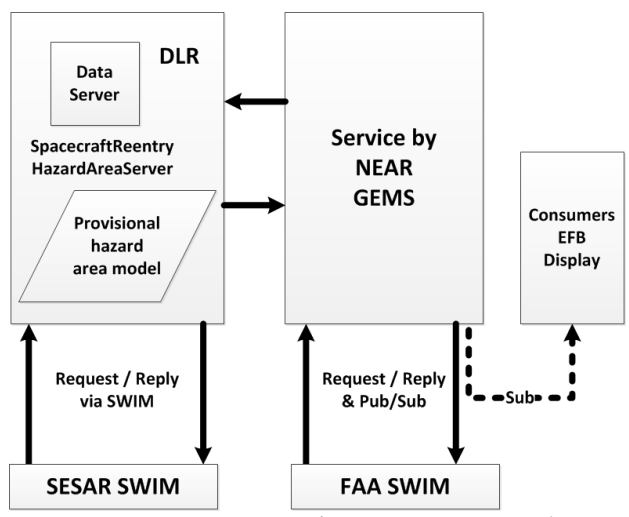

Figure 2. SESAR SWIM / FAA SWIM architecture

\subsection{Data Server}

The data server consists of an HTTPS web server, doing Simple Object Access Protocol (SOAP) message parsing for incoming requests and SOAP message creation for its responses. It benefits from using the following modules/extensions:

- Tcl httpd realizing the server side of the HTTP protocol handling and using Tcl's I/O channelling,

- $\quad$ Tls providing Secure Sockets Layer (SSL)-enabled TCP sockets in Tcl, supporting password protected SSL keys to improve security,

- WS::Sever exporting SOAP interfaces where HyperText Markup Language (HTML) page autogeneration, describing all methods and data types, is part of the service creation functionality,

- Rpcvar enabling complex data type definitions and their associated result packaging in the Remote Procedure Call (RPC) handler.

The service's reaction performance (server side time between receiving the request and sending out the response) is shown in Figure 3.

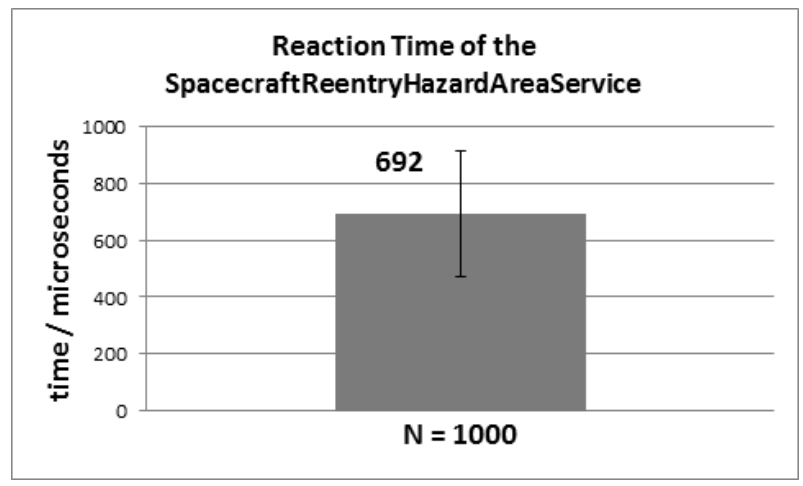

Figure 3. Reaction time of the SpacecraftReentryHazardAreaService

\subsection{Provisional Hazard Area Model}

The provisional hazard area model is currently characterized by a hard coded implementation of tabled 
debris data (derived from Columbia accident measurements), only representative for close to Space Shuttle like trajectory behaviour [1]. The relation in the tabled data can be summarized by:

$$
\text { Debris Area length }_{\text {in km }}=\frac{A l t_{\text {in feet }}}{1000}
$$

$$
\text { Debris Area width }=\frac{\text { Debris Area length }}{8}
$$

It must be emphasized, that these formula are not used, but one-dimensional table interpolation (first column is independent variable, all others dependent) is taken , facing the preparation for potential future tabled data of other vehicles, not reflecting a simple Space Shuttle flight dynamics based altitude-area specific case relationship as in (1) and (2), but taking into account state vector, velocity as well as vehicle specific aerodynamic coefficients data. The performance of the associated processing function is shown in Figure 4. Further, the hazard area calculation is prepared for:

- Lagrange interpolation for finding polynomial of degree $\mathrm{n}$ for a table of $\mathrm{n}$ sets of data,

- Neville algorithm usage for computing successively higher-degree approximations to the fit function by using the recurrence and take the straightest path possible,

- cubic spline interpolation for interpolating with different stepwise polynomials.

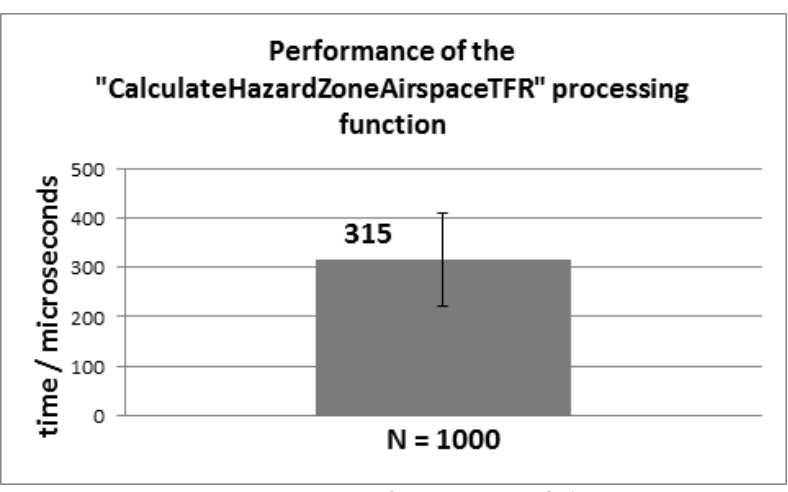

Figure 4. Performance of the

"CalculateHazardZoneAirspaceTFR" processing function using one-dimensional table interpolation

\section{OUTLOOK}

\subsection{Specific Hazard Area Model}

There is different research work going on referring the development of debris catalogues and deriving vehicle specific debris area data of finer granularity [3][4][5]. With a further development of the hazard area calculation model, a solution directly benefiting from newest data sets can be delivered in future, where a debris catalogues database connection will be established as shown in Figure 5.

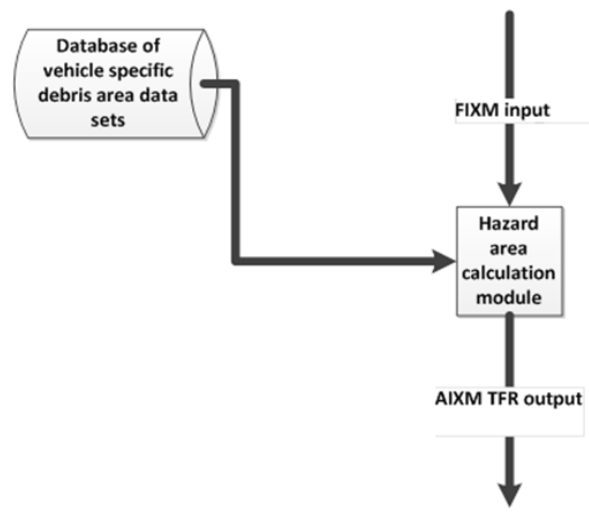

Figure 5. Vehicle specific hazard area calculation model

\subsection{Improving Scalability And Performance}

Tcl's underlying sophisticated I/O system multiplexes multiple clients without the complexities of threading. The service's reaction time performances for up to ten connected clients are shown in Figure 6. Each test ran for 1000 requests with a frequency of one client request per second.

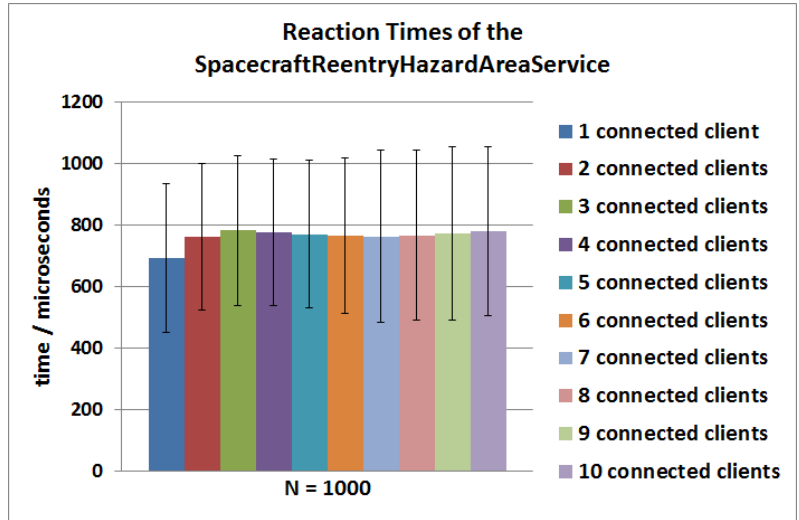

Figure 6. Reaction times of the SpacecraftReentryHazardAreaService

Because of the server's event driven $\mathrm{I} / \mathrm{O}$ handling in a non-blocking fashion, the maximum number of simultaneously served clients expecting response according to the request frequency is anticipated as:

$$
\text { clients }_{\max }=\frac{1 \text { ClientAverageFreq }_{\text {Re }_{\text {quest }}}}{\text { AverageReactionTime }_{\text {Service }}}
$$

Taking an average reaction time of 800 microseconds from Figure 6 and putting it in (3) with the tests' client request frequency of $1 \mathrm{~Hz}$ reveals a maximum number of 1250 simultaneous clients. Addressing the intension of the implementation of a vehicle specific hazard area model (5.1) in the future, the clients $s_{\max }$ number is 
estimated to be reduced by 30 to 50 percent, caused by needed database requests.

The following steps are planned to improve performance and scalability:

- Realization of a multi-server system with an on top application that directs clients to the appropriate servers,

- accompanying database split: a server only stores data related its subset of clients,

- full exploitation of Tcl 8.6's new Non-Recursive Engine (NRE) with its feature of supporting coroutines.

\subsection{Cyber-Security for SWIM}

Although the current prototype solution contains Transport Layer Security (TLS), it must be emphasized that this only secures point-to-point connections without intermediaries. Near future solution updates' developments will target to the implementation of message (end-to-end) security by using WS-Security technology throughout the whole chain of confidentiality, integrity, and availability as well as nonrepudiation. Leveraging technologies such as Security Assertion Markup Language (SAML), a service provider can authenticate appropriate users so that they can gain access to secure content. There remains the issue of availability in terms of system's resilience in presence of distributed denial of service (DDoS) attacks. DDoS attacks have been increasing in intensity and frequency lately. These attacks can knock of services provided by large service providers with ample resources. In order to address this threat, we suggest the necessary DDoS prevention techniques (filtering, load balancing, input sanitation, as well as traffic redirection) and technologies should be utilized to protect this system.

The measures for preventing spoofing will also be implemented. The necessary policies for issuing certificates and management (including revocation) need to be included as part of the WS-Security technology will be clearly defined and adapted according to re-entry needs. WS-Security adaptation will include use of Public Key Infrastructure (PKI) with appropriate certificate authority (CA) in order to assure the integrity of the system. The adapted WS-Security system will be up to date enough to avoid some salient vulnerabilities such as the Weak Diffie-Hellman and the Logjam Attack [2].

The solution is already prepared with server side "intransform" and "outtransform" options for further SOAP header injections' handling and processing, building the fundament for the future WS-Security as well as on top WS Trust implementation development.

\section{SUMMARY}

The paper presents the need of future commercial spacecraft being able to act as communicating subsystems in the system wide information management environment (SWIM). The air traffic integration challenge with the associated spacecraft specific properties is addressed as well as its global character's implications on ATM specific SWIM. A prototype solution with first test data results against an European / U.S. harmonization use case background is introduced with an outlook to future developments, covering precision, performance and security aspects.

\section{REFERENCES}

1. Robledo, L. F. (2004). Analysis and integration of a debris model in the VIRTUAL RANGE PROJECT. A thesis submitted in partial fulfillment of the requirements for the degree of Master of Science in Modeling and Simulation. Orlando, Florida: College of Engineering and Computer Science at the University of Central Florida.

2. Adrian, D., Bhargavan, K., Durumeric, Z., Gaudry, P., Green, M., Halderman, J.A., Heninger, N., Springall, D., Thomé, E., Valenta, L., VanderSloot, B., Wustrow, E., Zanella-Béguelin, S., Zimmermann, P. (2015). Imperfect Forward Secrecy: How DiffieHellman Fails in Practice. In Proc. $22^{\text {nd }} A C M$ Conference on Computer and Communications Security, Denver, CO, USA.

3. Ailor, W., Wilde, P. (2008). Requirements for warning aircraft of reentering debris. In Proc. $3^{\text {rd }}$ IAASS 'Building a Safer Space Together', European Space Agency, Rome, Italy.

4. Sarconi, M. (2013). A prototype system for simulating the risks of sub-orbital space flight for commercial aviation, Level 4 Project, School of Computing Science University of Glasgow, Glasgow, UK.

5. Falsone, A., Noce, F. \& Prandini, M. (2014). A randomized approach to space debris footprint characterization. In Proc. $19^{\text {th }}$ World Congress The International Federation of Automatic Control, Cape Town, South Africa.

6. Morlang, F., Hampe, J., Kaltenhaeuser, S., Jakobi, J., Schmitt, D.-R.. (2014). Commercial Space Transportation and Air Traffic Insertion - SESAR Requirements and the European Perspective. In Proc. 1st Annual Space Traffic 
Management Conference, Daytona Beach FL, USA.

7. Seker, R. Moallemi, M., Yapp, J., Towhidnejad, M., and Klein, R. (2014) "Performance Issues in Aircraft Access to the National Airspace SWIM Program" Integrated Communications Navigation and Surveillance Conference, Herndon, VA. 